\title{
Frieze Patterns of Integers
}

\author{
Karin BaUr
}

1 first encountered John Conway's work on frieze patterns around 2006 when I participated in a reading group on cluster algebras and related topics in the pure mathematics group at the University of Leicester. The two papers $[7,8]$ were on our reading list at the time, and they are most remarkable, for they invite the reader to a discovery of the properties of frieze patterns. The first presents a list of 39 problems to study, whereas the second gives hints and solutions for them. Problems (28) and (29) are known as the Conway-Coxeter theorem (Theorem 1 below), relating integer friezes with triangulations of polygons:

Problem (28): Is there just one frieze pattern of integers for each triangulated polygon?

Problem (29): Is there just one triangulated polygon for each frieze pattern of integers?

These two problems have led to a huge amount of activity around friezes, and in this article, I provide an overview, not exhaustive to be sure, of the development initiated by Conway's contribution to friezes almost 50 years ago. It goes without saying that there is not enough space here to give credit to all of the developers of this area. There are currently 88 articles on MathSciNet with the word "frieze" in the title. Some of them appeared in the 20 years following the inception of friezes. However, the discovery of the link between friezes and cluster algebras by Fomin and Zelevinsky [10] and between friezes and cluster categories by Caldero and Chapoton [6] led to renewed interest in the topic, with over 60 articles having been written on friezes in the last ten years.

Frieze patterns were invented by Coxeter in [9], where he attributed the first instance of such a frieze pattern to relations Gauss proved in his study of the pentagramma mirificum, a spherical pentagram formed by five successively orthogonal great-circle arcs. This gives a pattern as follows, arising from the 5-cyclic recurrence $\left(u_{i}\right)_{i}$ with $u_{i-1} u_{i+1}=1+u_{i}$, for all $i$ (reducing indices modulo 5):

$\begin{array}{ccccccccccccc} & & 0 & & 0 & & 0 & & 0 & & 0 & \\ & 1 & & 1 & & 1 & & 1 & & 1 & & \\ \ldots & & u_{1} & & u_{3} & & u_{5} & & u_{2} & & u_{4} & \\ & u_{5} & & u_{2} & & u_{4} & & u_{1} & & u_{3} & & \cdots \\ & 1 & & 1 & & 1 & & 1 & & 1 & \\ & & & 0 & & 0 & & 0 & & 0 & & \end{array}$

Coxeter also related this first example to continued fractions by considering $c_{i}=1+u_{i}$, and he proved further that frieze patterns of integers are characterized by a divisibility condition on entries in a diagonal.

Coxeter's original definition [9] has been generalized in many directions, resulting in work on infinite friezes, $\mathrm{SL}_{k}$-friezes, $\mathrm{SL}_{k}$-tilings, tropical friezes, 2-friezes, $q$-deformed friezes (see Ovsienko and Morier-Genoud's article in this issue [13]), etc. It became apparent that friezes are of interest in various areas such as combinatorics, cluster algebras, geometry, integrable systems, and representation theory. For a good survey, see Sophie Morier-Genoud's paper [12], in which many of these directions and links to the literature can be found. Frieze patterns were the topic of a mini-workshop in Oberwolfach in 2015 [17]; an MFO snapshot authored by Thorsten Holm appeared subsequently [11]. The combinatorial flavor of friezes has also made them quite popular for outreach activities. There are even two beautiful videos on friezes by Numberphile, presented by Sergei Tabachnikov [15, 16].

Here, I concentrate on the combinatorial geometric interpretation of friezes inspired by Problems (28) and (29) above. After recalling the notion of a frieze pattern and giving some of the properties of closed friezes, I concentrate on infinite friezes, offering a geometric interpretation 
via matchings and explaining the growth of the entries in those friezes.

\section{Frieze Patterns}

First, some definitions.

Definition 1. A frieze pattern is formed by a finite or infinite number of rows, shifted with respect to each other, starting with a row of 0's, followed by a row of 1's and then rows of elements $a_{i j}, i+2 \leq j$, of an integral domain (here, the integral domain will usually be the integers, but there will be an instance in which the entries are cluster variables of a cluster algebra):

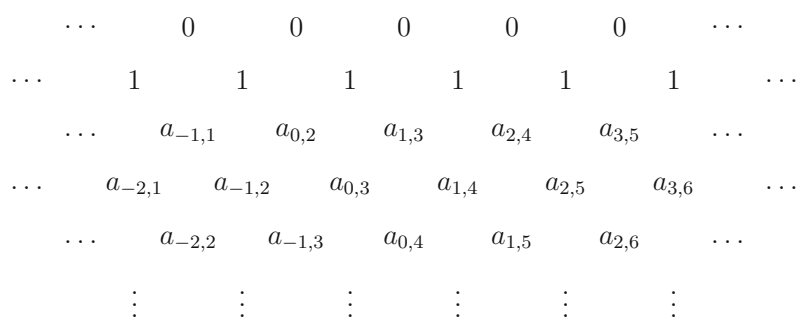

There are two conditions on these patterns. First, every square formed by four neighboring entries must satisfy the unimodular or diamond rule: we have $b c-a d=1$ for every four entries of the form

$$
b^{c}{ }^{c}
$$

Second, such a pattern must be periodic: there is an $n>0$ such that $a_{i, j}=a_{i+n, j+n}$, for all $i+2 \leq j$.

A frieze pattern is called integral if all entries $a_{i j}$ are positive integers. It is called closed if after a finite number of rows, it stops with a second row of 1's followed by a row of 0's. Otherwise, the frieze pattern is infinite. The order of a closed frieze is defined to be one less than its number of rows (including the zeros). (The number of nontrivial rows is called the width of the frieze.) An example of a closed integral frieze of order 6 appears in Figure 1. An example of an infinite frieze will be given below in Example 3.

Every integral frieze pattern is determined by its first nontrivial row. This is true more generally (for integer domains) if every $3 \times 3$ diamond in the frieze has determinant 0 . Such frieze patterns are said to be tame. If the frieze is $n$-periodic and tame, then any $n$ successive entries $a_{i, i+2}, \ldots, a_{i+n-1, i+n+1}$ determine the frieze under iterated application of the diamond rule. Such a tuple is called a quiddity sequence for the frieze.

From now on, we will mostly concentrate on integral friezes. In this case, we have a geometric interpretation of frieze patterns via triangulations of polygons (if the frieze

$\begin{array}{ccccccccccccc}0 & & 0 & & 0 & & 0 & & 0 & & 0 & & 0 \\ & 1 & & 1 & & 1 & & 1 & & 1 & & 1 & \\ & & 1 & & 4 & & 1 & & 2 & & 2 & & 2 \\ & 1 & & 3 & & 3 & & 1 & & 3 & & 3 & \\ 1 & & 2 & & 2 & & 2 & & 1 & & 4 & & 1 \\ & 1 & & 1 & & 1 & & 1 & & 1 & & 1 & \\ 0 & & 0 & & 0 & & 0 & & 0 & & 0 & & 0\end{array}$

Figure I. A closed integral frieze of order 6.

pattern is closed) or of annuli (if it is infinite), as we will see.

\section{Closed Frieze Patterns}

First note that if $\mathcal{F}$ is a closed integral frieze of order $n$, then $\mathcal{F}$ is $n$-periodic; cf. [7, (21)]. This can be viewed as a consequence of the following theorem, known as the ConwayCoxeter theorem.

Theorem 1. [7, 8, Problems (28), (29)] There is a bijection between frieze patterns of order $n$ and triangulations of convex $n$-gons.

To see how this works, consider a convex polygon $P_{n}$ with $n$ vertices, labeled clockwise by $\{1,2, \ldots, n\}$. Take a triangulation of $P_{n}$, i.e., a maximal collection by noncrossing diagonals of $P_{n}$ (any such collection contains $n-3$ diagonals). Let $a_{i}$ be the number matchings of triangles with vertex $i$, i.e., the number of triangles incident with vertex $i$. Then $\left(a_{1}, \ldots, a_{n}\right)$ is the quiddity sequence of a frieze pattern of $n$.

One of the first properties of frieze patterns is that every quiddity sequence of a closed integral frieze contains at least two entries equal to 1 . Theorem 2 gives a geometric reason for this, since every triangulation contains at least two triangles that are peripheral, i.e., for which two edges are boundary segments. Another fact is that if $\left(a_{1}, \ldots, a_{n}\right)$ is a quiddity sequence with $n>2$, then there can be no two entries 1 next to each other.

REMARK 1. There is a way to go from quiddity sequences with $n$ elements to quiddity sequences with $n+1$ elements and back, as described in [7, (23)]: If $\left(a_{1}, \ldots, a_{n}\right)$ is the quiddity sequence of a frieze pattern of order $n$ and $i$ is some index, $1 \leq i \leq n$, then the sequence $\left(a_{1}, \ldots, a_{i}+\right.$ $\left.1,1, a_{i+1}+1, \ldots, a_{n}\right)$ is the quiddity sequence of a frieze pattern of order $n+1$. We call this operation "gluing." The reverse operation of gluing is to start with an entry $a_{i}=1$ in a quiddity sequence and remove it, by simultaneously decreasing its two neighbors by 1: from $\left(a_{1}, \ldots, a_{i-1}, 1, a_{i+1}, \ldots, a_{n}\right)$, we obtain the new quiddity sequence $\left(a_{1}, \ldots, a_{i-1}-1, a_{i+1}-1, \ldots, a_{n}\right)$, with $n-1$ entries. This is called "cutting (at an entry 1)."

In terms of triangulations of polygons, gluing corresponds to adding a vertex with a peripheral triangle to the 
triangulated polygon, while the operation cutting corresponds to removing a peripheral triangle.

REMARK 2. (Idea of the proof of Theorem 1: first, check that the claim is true for $n=3$, and then use induction.) To go from a frieze pattern $\mathcal{F}$ of order $n \geq 2$ to a triangulated $n$-gon, modify the quiddity sequence of $\mathcal{F}$ by cutting at an entry 1 . The result is a frieze pattern $\mathcal{F}^{\prime}$ of order $n-1$. By the induction hypothesis, this corresponds to a triangulation of a polygon on $n-1$ vertices. Glue a triangle at the appropriate position to get the desired triangulation.

To go from a triangulated $n$-gon with $n \geq 4$ to a frieze, use the fact that every triangulated polygon has a peripheral triangle. Removing this gives a triangulated polygon with $n-1$ vertices. This corresponds by induction to a closed frieze of order $n-1$. Extending its quiddity sequence by gluing yields the quiddity sequence for the desired triangulated $n$-gon.

By Theorem 1, we can give frieze labels of the vertices of the polygon to the entries of any closed integral. The entries in the quiddity sequence $\left(a_{n, 2}, a_{1,3}, \ldots, a_{n-1,1}\right)$ are viewed as being in positions $(n, 2),(1,3),(2,4), \ldots,(n-1,1)$, and by going along a diagonal NW-SE, we keep the first coordinate, with the entries in diagonals NE-SW having the second entry fixed. Figure 2 shows this for $n=6$.

With this labeling system, we can explain how every entry in a closed integral frieze is given by a set of matchings for a triangulation, a result of Broline, Crowe, and Isaacs [5].

Consider a triangulated convex $n$-gon $P$. Let $i, j$ be two vertices of $P$ with $j \notin\{i-1, i, i+1\}$. Denote by $\mathcal{M}(i, j)$ the set of matchings of triangles with the set of vertices $\{i+$ $1, \ldots, j-1\}$ (reducing modulo $n$ ). Note that

$$
\{i+1, \ldots, j-1\} \cup\{j+1, \ldots, i-1\}=\{1, \ldots, n\} \backslash\{i, j\} .
$$

Theorem 2. [5, Theorem 1] Let $T$ be a triangulation of a convex $n$-gon $P$ and let $\mathcal{F}=\left(a_{i j}\right)_{i j}$ be the frieze associated with $T$. Then $|\mathcal{M}(i, j)|=a_{i j}=|\mathcal{M}(j, i)|$ for any two vertices $i, j$ of $P$ with $j \notin\{i-1, i, i+1\}$.

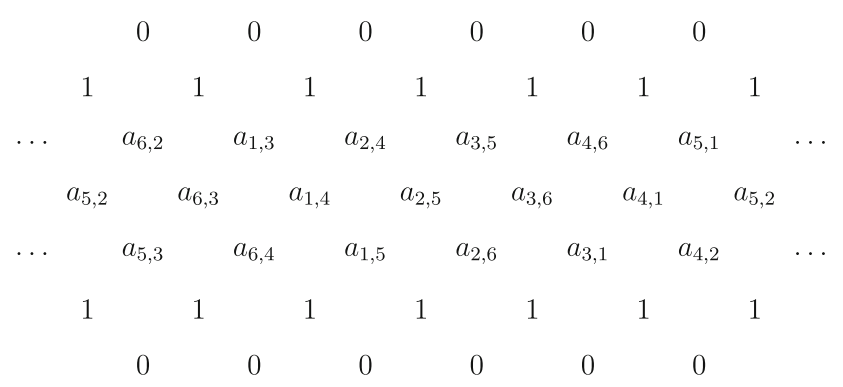

Figure 2. The layout of a frieze of order 6 .
Example 1. Consider a fan triangulation of a hexagon with all diagonals incident with vertex 1 , as shown in Figure 3 .

The quiddity sequence of the corresponding frieze is (4, 1, 2, 2, 2, 1), as depicted in Figure 1. To illustrate Theorem 2, we determine a few sets of matchings. There are four triangles incident with vertex 1 ; the set of matchings for vertex 1 is $\mathcal{M}(6,2)=\left\{\Delta_{1}, \Delta_{2}, \Delta_{3}, \Delta_{4}\right\}$ and $a_{6,2}=4$.

For entry $a_{6,3}$, we have to consider matchings between triangles and the vertices 1,2 :

$$
\mathcal{M}(6,3)=\left\{\left(\triangle_{1}, \triangle_{4}\right),\left(\triangle_{2}, \triangle_{4}\right),\left(\triangle_{3}, \triangle_{4}\right)\right\}
$$

(the first entry in the tuple is the triangle allocated with vertex 1 ; the second entry is the triangle allocated with vertex 2$), a_{6,3}=3$. We compare this with $\mathcal{M}(3,6)$, matching triangles to the vertices 4,5 of the polygon:

$$
\mathcal{M}(3,6)=\left\{\left(\triangle_{3}, \Delta_{2}\right),\left(\Delta_{3}, \Delta_{1}\right),\left(\Delta_{2}, \Delta_{1}\right)\right\}
$$

and $a_{3,6}=3$.

Let $\mathcal{F}$ be a closed integral frieze. Let $n_{0}$ be its minimal period. If $\mathcal{F}$ is finite of order $n$, it arises from a triangulation of an $n$-gon. The minimal period $n_{0}$ is equal to $n / 3$ if the triangulation is invariant under a rotation of 120 degrees, and is equal to $n / 2$ if the triangulation is invariant under a rotation of 180 degrees. It is equal to $n$ otherwise.

Example 2. Consider the closed friezes of small order. If $\mathcal{F}$ is of order 4 , its quiddity sequence is $(1,2,1,2)$ or $(2,1,2,1)$. In both cases, the smallest period is 2 . If $\mathcal{F}$ has order 5 , it arises from a triangulation of a pentagon, and since no such triangulation has a nontrivial rotational symmetry, the smallest period is 5 . If $\mathcal{F}$ has order 6 , it comes from a triangulation of a hexagon. Among such triangulations are the triangulations with rotational symmetry by 180 degrees, e.g., with diagonals $(1,3),(3,6),(4,6)$, and the triangulations with 3-fold symmetry, e.g., with diagonals $(1,3),(1,5)$, and $(3,5)$. In the former case, the frieze has period 3, while in the latter case, it is 2-periodic.

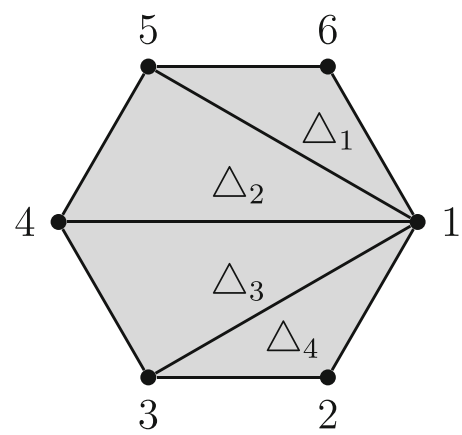

Figure 3. Fan triangulation of a hexagon with all diagonals incident with vertex 1 . 
In Figure 4 we show the 5-periodic frieze of order 5 , with quiddity sequence $(1,2,2,1,3)$, and a 2-periodic frieze of order 6 , with quiddity sequence $(1,3,1,3,1,3)$. For later use, we add rows of -1 's at both ends.

\section{A Frieze Determinant}

As an immediate consequence of the geometric interpretation by Broline et al. [5] of all frieze entries, we recover the $n$-periodicity of friezes of order $n$, and we see that closed integral friezes are invariant under a glide reflection. We consider a fundamental domain for this glide reflection given by the entries in positions $(i, j)$ with $1 \leq i \leq n-3$ (as in Figure 2). We include the $n-1$ entries equal to 1 and the $n$ entries equal to 0 above as well as the entry 1 below the two positions $(1, n-1)$ and $(2, n)$. These entries form a triangle, and we take it as the upper triangular part of an $n \times n$ matrix, with a row of 0's on the diagonal. Reflecting along the diagonal, we create a symmetric matrix $M=$ $M(T)$ whose entries are the matching numbers of a frieze pattern. This matrix depends on the chosen triangulation or on the corresponding matching numbers. Its determinant, however, is independent of these choices; it depends only on the size of the polygon.

Theorem 3. [5, Theorem 4] Let $M$ be the symmetric matrix corresponding to the frieze of a triangulation of an $n$-gon. Then $\operatorname{det} M=-(-2)^{n-2}$.

The determinant result can be stated in terms of cluster variables. We associate the entries of a frieze of order $n$ with the diagonals of a convex $n$-gon. These, in turn, correspond to cluster variables $x_{i j}, 1 \leq i<j-1 \leq n$, of a cluster algebra of type $\mathrm{A}_{n-3}$; see [10, Section 12.2]. The clusters of this cluster algebra are given by the triangulations of the $n$-gon, together with the frozen variables $\left\{x_{1,2}, x_{2,3}, \ldots, x_{n-1, n}, x_{n, 1}\right\}$ of the edges of the polygon: $\left\{x_{i j} \mid(i, j)\right.$ is a diagonal of $\left.T\right\} \cup\left\{x_{i, i+1} \mid i=1, \ldots, n\right\}$.

The variables of the diagonals in the chosen triangulation are the initial cluster variables. Every cluster variable of the associated cluster algebra is a Laurent polynomial in the initial variables, obtained through iterated mutations (a procedure similar to the diamond rule). Furthermore, the mutable cluster variables correspond bijectively to the diagonals in the $n$-gon. We put these variables in the positions as indicated by the endpoints of their diagonals. Then the cluster variables form a "frieze" $\mathcal{F}=\mathcal{F}(\underline{x})$ of elements of a ring of Laurent polynomials, in particular, of an integral domain. This is a slight generalization of the original definition of frieze patterns, replacing the two rows of 1 's by the frozen variables $\left\{x_{1,2}, x_{2,3}, \ldots, x_{n-1, n}, x_{n, 1}\right\}$ and naturally extending the labeling system with endpoints of diagonals in the $n$-gon. We note that $\mathcal{F}(x)$ is tame.

As before, in the resulting closed frieze, there is a fundamental domain given by the $x_{i j}$ with $1 \leq i \leq n-1$ and $i+1 \leq j \leq n$. We use this fundamental domain as the upper triangular part of a square matrix $M=M(\underline{x})$ whose entries are cluster variables and make it symmetric around the diagonal of 0's.

Theorem 4. [3, Theorem 3.2] Let T be a triangulation of a convex n-gon with initial cluster $\underline{x}=\underline{x}(T)=\left\{x_{i j} \mid(i j) \in\right.$ $T\} \cup\left\{x_{i, i+1}\right\}_{i}$. Let $\mathcal{F}(\underline{x})$ be the frieze of all the cluster variables obtained from this cluster and let $M(\underline{x})$ be the square matrix of $\mathcal{F}(\underline{x})$. Then

$$
\operatorname{det} M(\underline{x})=-(-2)^{n-2} x_{12} x_{23} \cdots x_{n-1, n} x_{n 1} .
$$

If we specialize the cluster variables $x_{i j}$ with $(i j) \in T$ and the frozen cluster variables to 1 , then the entries in the fundamental domain become the entries of the closed integral frieze pattern of $T$ [6, Proposition 5.2], and the frieze determinant from Theorem 4 specializes to the frieze determinant from Theorem 3.

REMark 3. It would be interesting to see whether an analogous formula involving frozen variables can be found for cluster algebras in type $D_{n}$ that arise from triangulations of a punctured disk.

\section{Infinite Friezes}

The Conway-Coxeter theorem tells us how quiddity sequences of finite integral friezes arise. It is natural to ask which sequences yield infinite integral frieze patterns. An example that has already appeared in [7, Problem (16)] is the one on the right in Figure 5. In [18], Manuela Tschabold showed that such patterns arise from triangulations of punctured disks. Let $S_{n}$ be a disk with $n$ marked points on the boundary and a point in the middle (the puncture), and

\begin{tabular}{|c|c|c|c|c|c|c|c|c|}
\hline-1 & -1 & & -1 & & -1 & -1 & & -1 \\
\hline 0 & & 0 & & 0 & & 0 & 0 & \\
\hline 1 & 1 & & 1 & & 1 & 1 & & 1 \\
\hline 1 & & 3 & & 1 & & 3 & 1 & \\
\hline 2 & 2 & & 2 & & 2 & 2 & & 2 \\
\hline 3 & & 1 & & 3 & & 1 & 3 & \\
\hline & 1 & & 1 & & 1 & 1 & & 1 \\
\hline 0 & & 0 & & 0 & & 0 & 0 & \\
\hline-1 & -1 & & -1 & & -1 & -1 & & -1 \\
\hline
\end{tabular}

Figure 4. The minimal period of the left-hand frieze is equal to its order. On the right, the minimal period is 2 , and the order is 6 . The rows of -1 's are added for our later discussion of growth coefficients. 
use $n$ arcs to triangulate $S_{n}$. These arcs have as endpoints the vertices on the boundary or the point in the middle, and they are noncrossing (the arcs are considered up to isotopy fixing endpoints). The resulting regions have three sides in general; two of them may coincide. For each vertex $i$ of the boundary, we let $a_{i}$ be the number of connected components of the complement of the triangulation in a small neighborhood of $i$.

Theorem 5. [18, Theorem 3.6]. If $a_{i}$ is given by a triangulation of the punctured disk $S_{n}$, then $\left(a_{1}, \ldots, a_{n}\right)$ is the quiddity sequence of an infinite n-periodic integral frieze.

Furthermore, as in the finite case, every entry in the frieze of a triangulation of $S_{n}$ is a matching number between triangles and vertices (on passing to a suitable covering of the punctured disk); see [18, Section 4.5].

ExAmple 3. Consider the star triangulation of a punctured disk with four vertices on the boundary, as shown on the left in Figure 5. At each vertex on the boundary, there are two triangles. We thus have $a_{i}=2$ for all $i$. The frieze associated with the quiddity sequence $(2,2,2,2)$ appears on the right in that figure.

Tschabold's results show that there are infinite friezes for every period $n>0$ and that the infinite friezes constructed from triangulations of $S_{n}$ have a geometric interpretation as in the case of closed friezes. However, these are by no means the only types of infinite friezes. It can be shown that if $\mathcal{F}$ is an infinite $n$-periodic frieze with quiddity sequence $\left(a_{1}, \ldots, a_{n}\right)$, then for all $b>0$, the $n$-tuple $\left(a_{1}+\right.$ $\left.b, a_{2}, \ldots, a_{n}\right)$ is also the quiddity sequence of an infinite $n$ periodic frieze [4, Theorem 2.2]. Even if the original sequence can be realized by a triangulation of $S_{n}$, this is no longer true for the new quiddity sequence.

However, every quiddity sequence of an infinite frieze can be realized as the matching numbers of a triangulation of an annulus. Consider an annulus with $n$ points on the outer boundary and $m$ points on the inner boundary, assuming that $n+m>0$. A triangulation of this surface is a collection of $n+m$ arcs connecting these points so that no two arcs cross. Again, we take arcs up to isotopy fixing endpoints. See Figure 6 for two examples of triangulations of annuli. A triangulation of an annulus gives rise to two quiddity sequences in general (only one if one of the boundaries has no marked points), similarly as in the case of triangulations of $S_{n}$ : if the outer boundary has $n>0$ vertices, we let $a_{i}$ be the number of connected components of the complement of the triangulation in a small neighborhood of $i$. One can show that the tuple $\left(a_{1}, \ldots, a_{n}\right)$ is the quiddity sequence of an infinite frieze. Furthermore, every infinite frieze arises in this way, as formalized in the following theorem.

Theorem 6. [4, Theorem 4.6] Every infinite periodic frieze comes from a triangulation of an annulus. All entries of the frieze are matching numbers between triangles and sets of vertices.

REMARK 4. The proof of Theorem 6 is an explicit construction of a triangulation for a quiddity sequence $\left(a_{1}, \ldots, a_{n}\right)$ of an infinite frieze. This works as follows.

(1) If there exists an entry 1 among the $a_{i}$, reduce the quiddity sequence as in Remark 1 until there are no 1's left. Since the frieze is infinite, all entries in the quiddity sequence are greater than or equal to 2 after finitely many steps [4, Remark 4.7].

(2) Assume now that $a_{i} \geq 2$ for all $i$ and that there exists an entry $a_{i}>2$. Draw vertices $1,2, \ldots, n$ on the outer boundary of an annulus. Then add $a_{1}-1$ starting segments of arcs at vertex 1 . Next, put $a_{1}-1$ vertices on the inner boundary across vertex 1 and connect the starting segments to these vertices on the inner boundary. In a second step, draw $a_{2}-1$ starting segments of arcs at vertex 2 . The first of them is connected to the last vertex drawn for the arcs at vertex 1 . In addition, $a_{2}-2$ new vertices are drawn on the inner boundary. And so on. For vertex $n$, only $a_{n}-3$ new vertices are drawn on the inner boundary, since the last of the $a_{n}-1$ arcs at $n$ gets connected to the first vertex created for vertex 1 .

(3) If the quiddity sequence is $(2,2, \ldots, 2)$, then the frieze arises from a star triangulation of a punctured disk. Replace every arc of the star triangulation by an arc starting at the outer boundary of an annulus and spiraling around a

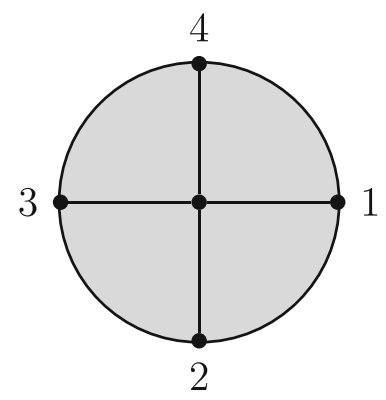

0

0

0

0 1

2

2

1

1

1

1

(3)

4

4

3

2

2

2

3

4

3

3

4

$4 \quad 4$

Figure 5. A triangulation of a punctured disk and its infinite integral frieze. 
noncontractible closed curve in this annulus (all in the same direction). See Example 4.

Example 4. The triangulation of an annulus giving rise to the trivial quiddity sequence $(2,2,2,2)$ is given by arcs spiraling around a noncontractible curve in the annulus, as shown on the left-hand side of Figure 6 . The triangulation yielding the quiddity sequence $(3,4,2,4)$ is shown on the right.

Note that we can view the triangulations of $S_{n}$ as coming from triangulations of an annulus with $m=0$ points on the inner boundary and $n$ points on the outer boundary, by contracting the inner boundary to a point.

\section{Growth of Frieze Patterns}

Clearly, in any closed frieze, the entries along a diagonal form a sequence of nonnegative integers that begins 0,1 , increases for a while, and then decreases back to 1 and 0 . If an infinite frieze arises from a triangulation of a punctured disk, it can be shown that the entries in every diagonal form $n$ arithmetic progressions [18, Proposition 3.12]. An illustration is the frieze pattern in Example 3. As Example 5 below suggests, the entries in an infinite frieze can grow much faster. In fact, we will see that in general, the numbers grow exponentially.

Example 5. The entries in frieze patterns arising from triangulations of annuli grow very fast. This is illustrated by the frieze with quiddity sequence $(3,4,2,4)$, as we see here. The entries in the fifth nontrivial row are already three-digit numbers:

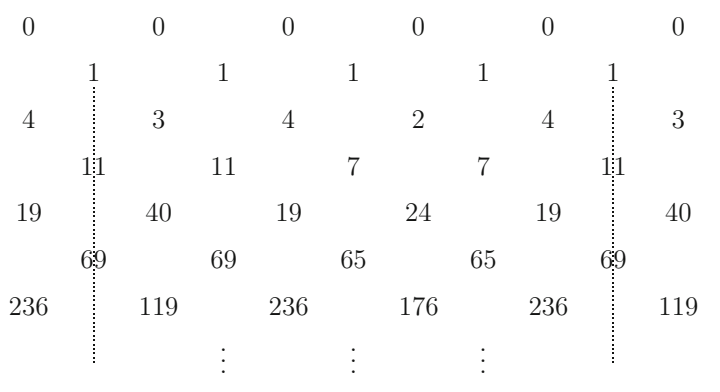

Recall that the elements in a frieze are indexed so that the entries in the quiddity sequence are of the form $a_{i, i+2}$. In going down a NW-SE diagonal, the first coordinate is fixed, while the second increases. One position to the right, both coordinates increase by 1 (Definition 1). Extend this and denote the entries in the first rows of 1 's by $a_{i, i+1}$, the entries in the rows of 0 's above by $a_{i, i}$, and the entries -1 above them by $a_{i, i-1}$. When drawing a frieze in the plane, we say that the (first) row containing the quiddity sequence is row 1 , and the rows above are called rows $0,-1$, and -2 (the last of these is the row of -1 's written above the frieze). If the frieze is closed of order $n$, then the frieze ends with a row of 1's (row $n-2$ ) followed by a row of 0 's (row $n-1)$, and we extend this by the additional row of -1 's (row $n$ ).

We can now state a remarkable property of integral friezes.

Theorem 7. [2, Theorem 2.2] Let $\mathcal{F}$ be an integral frieze. Assume that $\mathcal{F}$ is m-periodic. Then for all $k \geq 0$, the differences of the entries in rows $\mathrm{km}$ and $\mathrm{km}-2$ are constant: $a_{i, i+k m+1}-a_{i+1, i+k m}=a_{j, j+k m+1}-a_{j+1, j+k m}$ for all $i, j$.

There are two extreme cases in which the statement is clearly true: If $k=0$, the difference of the entries in rows 0 and -2 is always 2 . Secondly, if $\mathcal{F}$ is closed of order $n$, then it is in particular $n$-periodic. For closed friezes of order $n$, we reduce the coordinates of the entries modulo $n$, as indicated in Figure 2. So if $k=1$ and $m=n$, we have $a_{i, i+n+1}=a_{i, i+1}=-1, a_{i+1, i+n}=a_{i+1, i}=1$ for all $i$, with constant difference -2 . See Figure 4 for two examples.

These constant differences can be expressed using Chebyshev polynomials of the first kind. More precisely, for infinite friezes, the constant differences of entries occur every $n_{0}$ rows, where $n_{0}$ is the smallest period. We define $s:=a_{i, i+n_{0}+1}-a_{i+1, i+n_{0}}$ to be the first of these differences (apart from the one for $k=0$ ). In the frieze pattern of Example 3, we have $s=2$. In Example 5, we have $s=58$.

Note that if the frieze arises from a triangulation of a punctured disk, all these constants are equal to 2 (the first case to check here is the frieze in Figure 5). In all other infinite friezes, these differences grow rapidly. The sequence $\left(s_{k}\right)_{k \geq 0}$ of these constant differences satisfies the recurrence

$$
s_{k+2}=s_{1} s_{k+1}-s_{k} .
$$

Explicitly, in terms of $s=s_{1}$, we have

$$
s_{k}=s^{k}+k \sum_{l=1}^{\lfloor k / 2\rfloor}(-1)^{l} \frac{1}{k-l}\left(\begin{array}{c}
k-l \\
l
\end{array}\right) s^{k-2 l}
$$

for $k \geq 1$. Furthermore, the sequence $\left(s_{k}\right)_{k \geq 0}$ grows asymptotically exponentially if and only if $s>2$, in which case, its growth rate is $\frac{1}{2}\left(1+\sqrt{s^{2}-4}\right)$; see [2, Section 4] for
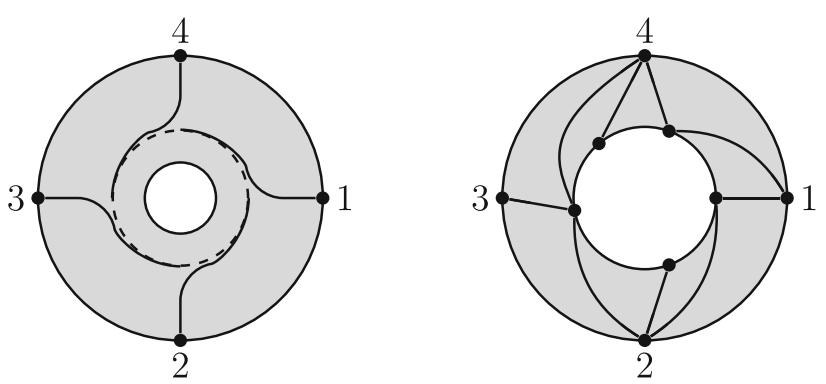

Figure 6. Triangulations for the quiddity sequences $(2,2,2,2)$ and $(3,4,2,4)$ 
details. Since $s$ governs the rate at which the entries in $\mathcal{F}$ grow, it is called the growth coefficient of the frieze.

REMARK 5. (1) Every triangulated annulus with marked points on both boundaries gives rise to two frieze patterns, since both boundary components determine a quiddity sequence. These sequences are different in general. However, one can show that their growth coefficients are the same [2, Theorem 3.4]. In the figure on the right in Figure 5, the quiddity sequence of the inner boundary is $(3,2,4,2,3)$, giving a 5-periodic frieze.

(2) Triangulations of annuli give rise to cluster tilting objects in a cluster category of type $\widetilde{A}$. In this algebraic interpretation, the common growth behavior of the two associated friezes can be viewed as common behavior of tubes in the associated category and interpreted as generalized numbers of submodules of indecomposable objects [1, Section 4.4]. However, there are examples of cluster categories with collections of tubes in which the growth coefficients differ across the different tubes.

Since my first encounter with Conway's contributions to frieze patterns, I have been fascinated by the many aspects of this topic. Several of the questions I have studied about these patters over the years link back to cluster algebras or cluster categories. Others are more combinatorial, such as the geometric realizations of infinite friezes. And some use homological methods, for example, in the reduction of friezes of Dynkin types to smaller friezes. Studying the growth of infinite friezes has interesting implications for module categories, as pointed out in Remark 5 above. Only recently, I learned that Conway thought about growth in a very different context, for the so-called look-and-say numbers, which he explained beautifully in a video by Numberphile from 2014 [14].

\section{ACKNOWLEDGMENTS}

I thank Sophie Morier-Genoud for helpful comments and suggestions. My work was supported by Royal Society Wolfson Fellowship 180004 and by FWF project P 30549. I also acknowledge support by FIM, ETHZ. I am currently on leave from the University of Graz, Austria.

Open Access This article is licensed under a Creative Commons Attribution 4.0 International License, which permits use, sharing, adaptation, distribution and reproduction in any medium or format, as long as you give appropriate credit to the original author(s) and the source, provide a link to the Creative Commons licence, and indicate if changes were made. The images or other third party material in this article are included in the article's Creative Commons licence, unless indicated otherwise in a credit line to the material. If material is not included in the article's Creative Commons licence and your intended use is not permitted by statutory regulation or exceeds the permitted use, you will need to obtain permission directly from the copyright holder. To view a copy of this licence, visit http://creativecommons.org/ licenses/by/4.0/.
Karin Baur

University of Leeds

Leeds

LS2 9JT

UK

e-mail: ka.baur@me.com

\section{REFERENCES}

[1] Karin Baur, Ilke Canakci, Karin M. Jacobsen, Maitreyee C. Kulkarni, and Gordana Todorov. Infinite friezes and triangulations of annuli. arXiv:2007.09411, 2020.

[2] Karin Baur, Klemens Fellner, Mark J. Parsons, and Manuela Tschabold. Growth behaviour of periodic tame friezes. Rev. Mat. Iberoam. 35:2 (2019), 575-606.

[3] Karin Baur and Bethany R. Marsh. Categorification of a frieze pattern determinant. J. Combin. Theory Ser. A 119:5 (2012), 1110-1122.

[4] Karin Baur, Mark J. Parsons, and Manuela Tschabold. Infinite friezes. European J. Combin. 54 (2016), 220-237.

[5] D. Broline, D. W. Crowe, and I. M. Isaacs. The geometry of frieze patterns. Geometriae Dedicata 3 (1974), 171-176.

[6] Philippe Caldero and Frédéric Chapoton. Cluster algebras as Hall algebras of quiver representations. Comment. Math. Helv. 81:3 (2006), 595-616.

[7] John H. Conway and Harold S. M. Coxeter. Triangulated polygons and frieze patterns. Math. Gaz. 57:400 (1973), 87-94.

[8] John H. Conway and Harold S. M. Coxeter. Triangulated polygons and frieze patterns. Math. Gaz. 57:401 (1973), 175-183.

[9] H. S. M. Coxeter. Frieze patterns. Acta Arith. 18 (1971), 297310.

[10] Sergey Fomin and Andrei Zelevinsky. Cluster algebras II: finite type classification. Invent. Math. 154:1 (2003), 63-121.

[11] Thorsten Holm. MFO Snapshot. Available online at https:// publications.mfo.de/handle/mfo/447, 2015.

[12] Sophie Morier-Genoud. Coxeter's frieze patterns at the crossroads of algebra, geometry and combinatorics. Bull. Lond. Math. Soc. 47:6 (2015), 895-938.

[13] Sophie Morier-Genoud and Valentin Ovsienko. Quantum real numbers and $q$-deformed Conway-Coxeter friezes. Mathematical Intelligencer 43:2 (2021).

[14] Numberphile. Look-and-say numbers. Available online at https:// www.youtube.com/watch?v=ea7lJkEhytA\&list=UUoxcjq-8xIDTY p3uz647V5A, 2014.

[15] Numberphile. Frieze Patterns-Numberphile. Available online at https://www.youtube.com/watch?v=0mXz-NP-raY, 2019. (Accessed December 21, 2020.)

[16] Numberphile2. Frieze Patterns (extra)—Numberphile. Available online at https://www.youtube.com/watch?v=MJ1NAzpens4\&t= Os, 2019. (Accessed December 21, 2020.)

[17] Mathematisches Forschungsinstitut Oberwolfach. Mini Workshop: Friezes. Available online at https://www.mfo.de/ 
occasion/1545a/www_view, 2015. (Accessed December 21, 2020.)

[18] Manuela Tschabold. Arithmetic infinite friezes from punctured discs. arxiv:1503.04352, 2015.
Publisher's Note Springer Nature remains neutral with regard to jurisdictional claims in published maps and institutional affiliations. 